\title{
Flugzeugmessungen von Ozon und Vorläuferstoffen im Raum Berlin-Brandenburg
}

\author{
Prof. Dr. sc. nat. Bernd Stark
}

\section{Anlaß/Ziele}

Die Zunahme des bodennahen Ozons sowie die unter bestimmten meteorologischen Bedingungen immer häufiger auftretende Überschreitung des Schwellenwertes von $180 \mu \mathrm{g} / \mathrm{m}^{3}$ für Ozon haben unter Fachleuten, den politisch Verantwortlichen und in der Bevölkerung zu großem Interesse und einer lebhaften Diskussion dieser Problematik gefuihrt, weil hohe Ozonwerte einmal zu gesundheitlichen Beeinträchtigungen des Menschen führen und zum anderen einen schädlichen Einfluß auf das Wachstum bestimmter, auch für den Menschen wichtiger Pflanzenarten haben. Im Mittelpunkt der Diskussion steht immer wieder die Frage der Möglichkeiten und Maßnahmen zur Verminderung der Emission der Vorläuferstoffe für die Ozonbildung.

Aus diesem Grunde wurden von den Ländern Berlin und Brandenburg Mittel zur Verfügung gestellt, um in einem bis zum gegenwärtigen Zeitpunkt einmaligen Projekt umfangreiche Flugzeug- und Bodenmessungen durchführen zu können. Die Messungen haben das Ziel, einmal die Kenntnisse über den Zusammenhang zwischen Schadstoffemissionen, den meteorologischen Bedingungen, dem Schadstofftransport und der Ozonbildung zu verbessern, zum anderen mit Hilfe von photochemischen Modellrechnungen die Auswirkungen verschiedenster Maßnahmen, die zur Reduktion der Emission von Ozonvorläuferstoffen im Raum Berlin/Brandenburg, aber auch großräumig denkbar sind, unter verschiedenen konkreten Wetterbedingungen und damit nachvollziehbar zu simulieren. Die Meßdaten dienen dabei der Kalibrierung und Überpüfung der Modellergebnisse. Der Raum Berlin-Brandenburg mit dem verkehrsintensiven und stark industrialisierten Ballungszentrum Berlin in dem ansonsten industrieschwachen und für deutsche Verhältnisse relativ dünn besiedelten Land Brandenburg stellt eine einzigartige Möglichkeit dar, den Beitrag eines solchen Konzentrationspunktes zur Erhöhung des überregionalen Ozonniveaus in diesem Großraum zu untersuchen.

\section{Beteiligłe und Durchführung}

An der Durchführung der Messungen waren 9 Institutionen beteiligt, die größtenteils ihren Sitz in Berlin und Brandenburg haben. An 5 Meßtagen wurden mit 2 Motorseglern (einer der Schweizer Firma MetAir, einer vom Institut für Meteorologie der FU Berlin) am Vormittag und Nachmittag die Ozon-, Stickoxid- und Kohlenwasserstoffkonzentration (C2-C10) in ca. $500 \mathrm{~m}$ Höhe über
Grund sowohl im Umland als auch über der Stadt gemessen. Diese Höhe spielt für den horizontalen Transport des Ozons und der Ozonvorläuferstoffe eine wichtige Rolle. Dabei wurde die Stadt meist in einem geschlossenen Horizontalprofil umflogen. Ergänzt wurden die Horizontalsondierungen von vorher festgelegten Vertikalsondierungen und Stadtüberquerungen.

Zusätzlich zu den festen Bodenmeßstellen des Berliner und Brandenburger Meßnetzes waren 3 Luftgüte-Meßwagen ( 2 aus Brandenburg, 1 der Senatsverwaltung für Stadtentwicklung und Umweltschutz Berlin) im Umland mit wechselnden Positionen aufgestellt, um die Belastung der Luft am Boden vor und hinter der Stadt Berlin zu messen. Ergänzt wurden die Messungen u. a. durch Wetterballonaufstiege in der Stadt und im Umland (Berlin-Tempelhof, Kremmen und Lindenberg), mit denen neben vertikalen Temperatur- und Feuchteprofilen vor allem die Stärke und Richtung der Luftströmungen gemessen wurde. Darüber hinaus wurden Windprofiler-Messungen, Ozonsondenaufstiege und Bodenozonmessungen am MOL Lindenberg sowie SODAR-Messungen in der Stadt und am Rande der Stadt (Berlin-Adlershof) durch das Fraunhofer-Institut für Atmosphärische Umweltforschung (IFU) und das Institut für Meteorologie der FU Berlin durchgeführt. Auf dem Frohnauer Meßturm im Norden Berlins wurde ebenfalls durch das IFU in $328 \mathrm{~m}$ Höhe über Grund Wasserstoffperoxid $\left(\mathrm{H}_{2} \mathrm{O}_{2}\right)$ gemessen, und es wurden Luftproben zur Bestimmung der VOCKonzentration genommen sowie durch die Senatsverwaltung für Stadtentwicklung und Umweltschutz Berlin BTX-Messungen mit Hilfe eines Gaschromatographen durchgefuihrt.

Die Messungen erfolgten unter der Leitung der Technischen Fachhochschule Wildau im Rahmen einer Kampagne vom 23.-27. Juli 1994. Dieser Zeitraum wurde in einer Anfang Juli 1994 angelaufenen telefonischen Konferenz festgelegt, an der die Projektleitung, das Wetteramt Potsdam des DWD, das Institut für Meteorologie der FU Berlin und Vertreter von SenStadtUm Berlin beteiligt waren. Dieser Verfahrensweise machte es möglich, mit großer Wahrscheinlichkeit eine hinreichend lange, geeignete Wettersituation vorherzusagen und auszuwählen.

\section{Erste Ergebnisse}

Die Meßperiode vom 23.-27. Juli 1994 war gekennzeichnet durch eine stabile Hochdruckwetterlage mit steigenden Temperaturen und gut korrelierter steigender Ozon- 
Monatsgang der Bodenozonwerte Juli 1994

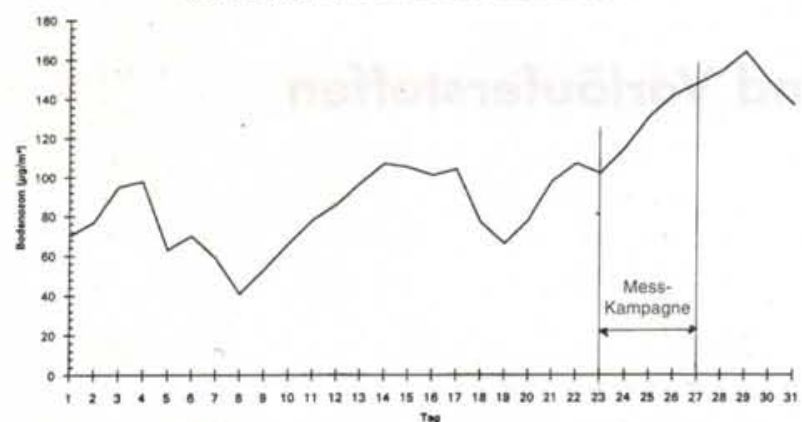

To

Abb. 1: Monatsgang der Bodenozonwerte, gemessen am MOL Lindenberg für Juli 1994. Der Meßkampagnenzeitraum ist markiert.

konzentration (Abb. 1). Der Wind hat im Verlauf der Meßkampagne von Ostsüdost über Süd nach Nordwest gedreht.

Die vorläufigen Auswertungen der umfangreichen Meßdaten ergeben, unabhängig von der Windrichtung, eine deutliche Erhöhung der Ozonkonzentration in der Abluftfahne der Stadt Berlin. Dieser Sachverhalt ist am Beispiel des 27.07.94 in den folgenden Abbildungen gezeigt. Während in der Stadt am Boden durch die Reaktion des Ozons mit den frisch emittierten Abgasen im Mittel 10-20\% weniger Ozon anzutreffen ist (Abb. 2),

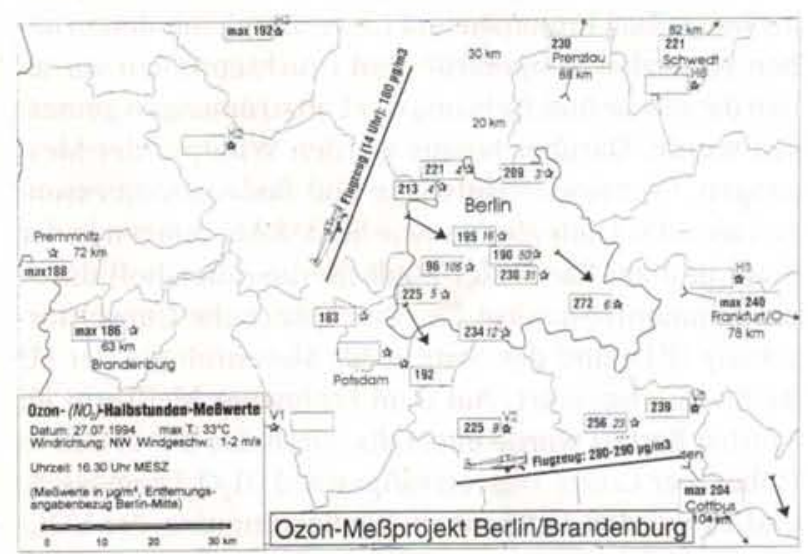

Abb. 2: Bodenmeßwerte von Ozon und Stickstoffdioxid für den 27.07.1994. In die Darstellung sind die Flugzeugmeßwerte in $500 \mathrm{~m}$ Höhe in Luv und Lee zum Vergleich eingezeichnet. Ozon: linker Wert im Rechteck. Stickstoffdioxid: rechter Wert im Rechteck.

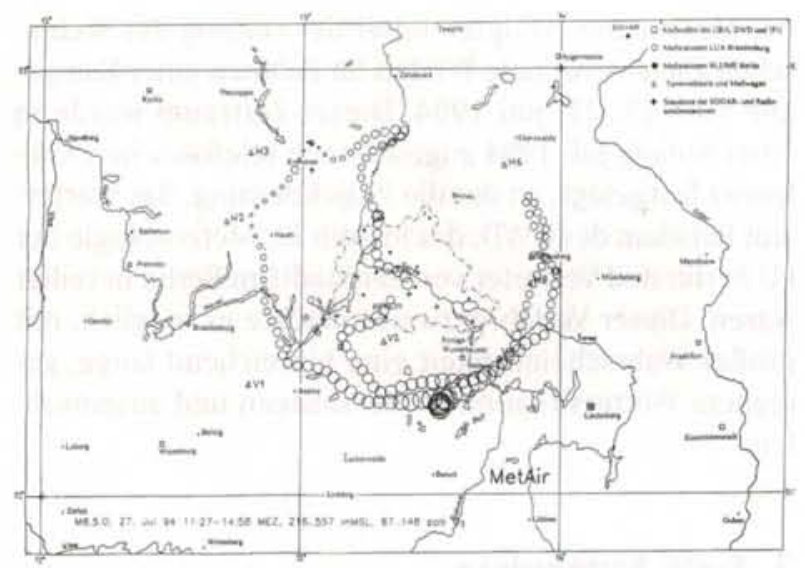

Abb. 3: Ozon-Flugzeugmeßwerte am 27.07.1994 in $500 \mathrm{~m}$ Höhe. Die Kreise stellen die Flugroute des Flugzeuges dar. Die Größe der Kreise ist ein Maß für die Ozonkonzentration. kleinster Kreis $\triangle 175 \mu \mathrm{g} / \mathrm{m}^{3}$ größter Kreis $\Delta 300 \mu \mathrm{g} / \mathrm{m}^{3}$ bildet sich zusätzliches Ozon auf dem Weg der Luft ins Umland. Im Gegensatz zum Boden ist in der Höhe während der Durchquerung der Emissionsgebiete von Berlin bereits ein linearer Anstieg der Ozonkonzentration zu beobachten. In 40-80 km Entfernung vom Zentrum Berlins war je nach Wettersituation eine Erhöhung der Lee-Konzentration gegenüber der Luv-Konzentration zwischen 20 und $50 \%$ zu verzeichnen. Vergleichbare Verhältnisse wurden für den gleichen Zeitraum auch in der Abgasfahne der Stadt München gefunden [1]. Die Abb. 3 zeigt für den 27.07.94 in einer Höhe von $500 \mathrm{~m}$ über Grund eine Erhöhung der Ozonwerte im Lee der Stadt um $50 \%$. Dieser Luv-Lee-Unterschied ist bis zu einer Höhe von $1500 \mathrm{~m}$ vorhanden, wie der in der Abb. 4 dargestellten Vertikalsondierung zu entnehmen ist.

Die Messungen werden ergänzt durch Modellrechnungen, die unter Verwendung aktueller Emissionsdaten sowie der im Rahmen der Meßkampagne gemessenen meteorologischen Parameter und der für den Start der Rechnungen notwendigen Initialisierungsdaten durchgeführt wurden. Das verwendete Simulationsmodell REGOZON wurde im Rahmen des DYMOS-Projektes im Institut FIRST der GMD Berlin entwickelt. Modellgrundlagen sind das mesoskalige Strömungs- und Transportmodell REWIMET [2] sowie der chemische Reaktionszyklus CBM-4 [3]. Eine berechnete Ozonverteilung ist für den 27.07.94 in der Abb. 5 dargestellt. Die Übereinstimmung der Modellrechnungen mit den gemessenen Daten ist auch in quantitativer Hinsicht beeindruckend.

Als vorläufiges Hauptergebnis des Projektes kann festgestellt werden, daß die Emissionen der Stadt Berlin die nachmittägliche Ozonspitzenkonzentration des Umlandes bei allen beobachteten Anströmrichtungen sehr deutlich - 20 bis $50 \%$ - erhöhen. Ein Anteil in dieser Größe konnte mit den bislang vorhandenen Meßstationen innerhalb der Stadt nicht festgestellt werden. Erst die beiden neu in Betrieb genommenen Meßstellen in Buch und am Müggelsee zeigten schon im Frühjahr 1994 aufgrund ihrer relativ großen Entfernung zu dicht besiedelten Stadtteilen mehr als die bislang diagnostizierten $20 \%$ Ozonzunahme.

Der in der Höhe durch die Flugzeugmessungen festgestellte Sachverhalt wurde durch die Messungen der Bodenmeßstellen bestätigt (Abb. 2).

\section{Schlußfolgerung}

Nach momentaner Einschätzung der Sachlage, abgeleitet aus den Meßergebnissen und den Modellrechnungen, kann gesagt werden, daß sich die Wirksamkeit von Geschwindigkeits- oder realistischen Verkehrsbeschränkungen auf weniger als 10\% Minderung der Gesamtbelastung an Ozon beschränkt. Insofern handelt es sich bei kurzfristigen adhoc-Maßnahmen dieser Art eher um solche mit politischer Signalwirkung, die in die richtige Richtung gehen, aber keinen wesentlichen Beitrag zur Problemlösung darstellen - es sei denn, es ließen sich Vorteile und damit Anreize für den Nutzer/Betreiber besonders abgasarmer Autos bzw. Anlagen koppeln. Jedenfalls sind emissionsmindernde Maßnahmen aufgrund der räumlichen Ausdehnung hoher Ozonwerte nur in einem 



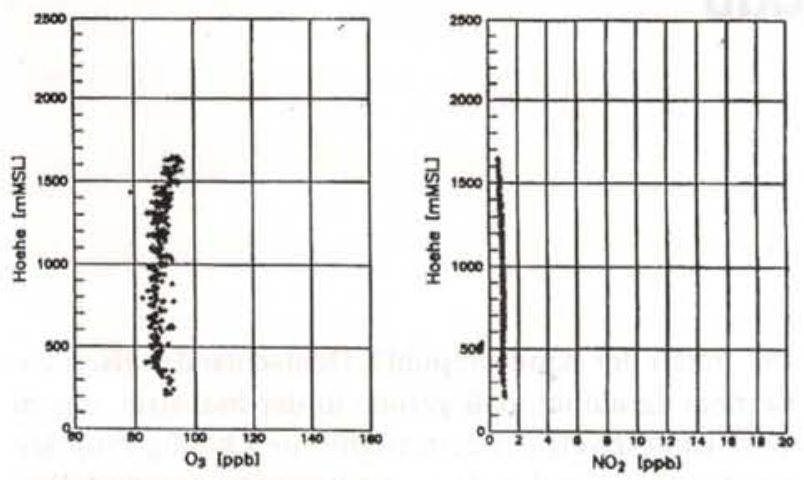

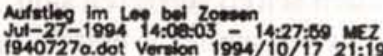


Abb. 4: Vertikalprofile von Ozon und Stickstoffdioxid für den 27.07.1994 im Luv und Lee von Berlin, gemessen in einer Vertikalsondierung.


Berechnete Ozonkonzentration in der $\mathrm{m}$-Schicht

Abb. 5: Berechnete Ozonkonzentration in der m-Schicht für den 27.07.1994. Simulationsmodell REGOZON [2]. Die Werte am Farbbalken stellen die Ozonkonzentration in $\mu \mathrm{g} / \mathrm{m}^{3}$ dar. gemeinsamen Handeln der Bundesländer Berlin und Brandenburg sinnvoll.

Eine deutlichere Senkung der Ozonbelastung wird vermutlich nur durch möglichst dauerhafte sowie regional und großräumig durchgeführte Emissionsminderungsmaßnahmen erzielbar sein, bei denen die Verläuferemissionen um $50 \%$ und mehr vermindert werden. Weiterführende Modellrechnungen werden helfen, diesen Sachverhalt genauer zu quantifizieren.

\section{Literatur}

[1] Greenpeace, Luft - aktuell

Ozonsmog - die Stadt als Ozonquelle, flugzeuggestiitzte Ozonmessungen und Ausbreitungsrechnungen im Ballungsraum München, Greenpeace e.V., Karsten Smid, September 1994

[2] Heimann, D. (1985)

Ein Dreischichten-Modell zur Berechnung mesoskaliger Windund Immissionsfelder über komplexem Gelände. Dissertation, Universität München

[3] Whitten, G.Z., Gery, M.W. (1986)

Development fo CBM-X mechanism for urban and regional AQSMs. US Environmental Protection Agency

\section{Verfasser}

Prof. Dr. sc.nat. Bernd Stark

Technische Fachhochschule Wildau Fachbereich Physikalische Technik Tel: (03375) 507194 